\section{DYNAMIC VIRTUAL MACHINE MIGRATION USING RATIO-BASED METHOD}

KEY WORDS: Cloud

Computing, Infrastructure as a Service, Energy Consumption, Virtual Machine.

\section{Dr. Haresh Damjibhai Khachariya}

Dr. Jayesh $\mathbf{N}$. Zalavadia*

\section{Assistant Professor, Shri M \& NVirani Science College, Rajkot}

Professor \& Head, Gyanyagna College of Science and Managment, Rajkot *Corresponding Author

Cloud computing provides various services over the internet and its increasing day by day. Given the growing demands of cloud services, it requires a lot of computing resources to meet customer needs. So, the addition of energy consumption through cloud computing resources will increase day by day and become a key obstacle in the cloud environment. In cloud computing, data centers consume more energy and additionally release carbon dioxide into the atmosphere. To reduce energy consumption through the cloud datacenter, energy-efficient resource management is required.

In this paper a specific technique for performing virtual machines through datacenter is given. Our goal is to reduce power consumption on the datacenter by reducing the host running in the cloud datacenter. To reduce power consumption, schedule the incoming task such a way that all the resources like ram, cpu(mips) and bandwidth utilize in equal weightage. Then after if any host is over utilized then migrate one or more vm from that host to another host as well as if any host is underutilize then migrate running vm of that host and switch off the under loaded host to save energy.

\section{INTRODUCTION}

Use of cloud resources are growing day by day and that's why the necessity of the computing resources are increase speedily. Thus for prime and economic performance cloud computing has become wide accepted approach. All the kind of IT facilities are provided by cloud computing to the user as a "service". Cloud computing, outlined as "a model for allowing convenient, on-demand network access to a shared pool of configurable computing resources which will be speedily allocated and deallocated with nominal administration effort or service provider interaction" in step with National Institute of Standards and Technology [1]. Cloud computing provides Infrastructure as a Service (IaaS) that offers infrastructure like, storage and resources to user for numerous purpose, Platform as a service(PaaS) provides platform to their shoppers for various application development and Software as a service(SaaS) that provides Software directly from the cloud systems [8]. Attractor of cloud computing is its elastic nature, service suppliers of cloud computing based mostly company may be elastic in their service delivery to satisfy the user needs. Give on demand services in pay-as-you-use manner.

Data centers are the backbone of the trendy economy. As report from NRDC, In 2013, U.S. information centers consumed estimated ninety one billion kilowatt-hours of electricity power, appreciate the annual output of thirty four giant (500megawatt) coal-fired power plants. Data-center electricity consumption is projected to extend to roughly a hundred and forty billion kilowatt-hours annually by 2020 , the equivalent annual output of fifty power plants, cost accounting American businesses $\$ 13$ billion annually in electricity bills and emitting nearly a hundred million metric plenty of carbon pollution annually.

These datacenters have typically created abundant less progress than their hyper-scale cloud counterparts owing to persistent problems and market barriers, like lack of metrics and transparency, and also misalignment of incentives.

For higher resource utilization and economic performance every physical machine contains numerous virtual machines that are used for efficient task scheduling. Logical partition of physical machine is thought as "virtual machine". VM is formed on physical machine and Cloudlets(tasks) submitted to VM. however in cloud computing systems running Physical machines consumes large quantity of energy and it'll produce energy shortage in future. Unorganized load over datacenter lead higher energy consumption. So, organize virtual machine over the host such a way that it minimize overall energy consumption in cloud data center. This paper uses novel approach for minimizing the ability usage of virtual machine. If virtual machine has no task then destroy that VM. Physical machine is under loaded then migrate that virtual machine and shutdown host to minimize the usage of energy. If host is overloaded then migrate virtual machine from overloaded host to other host. Virtual machine allocation is split into two ways. First is, either it'll use new virtual machine to manage load and second optimizing the present node. This method uses second methodology to allocation of virtual machine. This paper uses CPU, Memory and disc space parameter for energy optimization technique of virtual machine placement and proposed a new algorithm dynamic virtual machine migration using ratio based(DVMMRB).

\section{RelatedWorks}

Yukinori Sato and Yasushi Inoguchi[2] have proposed a green scheduling algorithm to reduce the energy consumption. They have used capacity of server to assign the jobs and based on workload they shift the states of host. They used concept of neural predictor to predict the upcoming workload based on earlier data. In scheduling, neural network is used to guess task load for data mapping on dynamic resources.

Jungwook Cho, Nakku Kim and Euiseong [3] proposed an Energy credit scheduling algorithm, in which energy credit value of each VM task is assigned to that machine to improve the energy optimization and reduce infrastructure cost. But they consider only processor energy consumption.

Albert P. M. De La Fuente Vigliotti and Daniel Mac edo Batista [4] have proposed an algorithm for placement of new virtual machine rather than old one. Knapsack strategy and Evolution Computation strategy are used for migration of VM allocation and management of tasks.

Liang Luo andWenjunWu[5] have proposed an algorithm for a cloud computing environment that could automatically allocate resources based on energy optimization methods. The classification of the job is divided into two parts. In the 
first part, before executing the job, user could select the type of his/her job, such as data-intensive or compute-intensive, memory intensive, network-intensive and I / O intensive. In the second step, when the job starts to execute, it will apply some method to test the type of jobs by counting the instruction execution speed in the CPU.

Yi-Ju Chiang, Yen-Chien Ouyang and Ching-Hsien Hsu [6] have proposed an algorithm that allows cloud providers to decide switch the mode of machine without sacrificing an SLA constrains. Resources in idle mode consume more energy. So, turned off idle computers for power saving without violating the service level agreement (SLA). It reduces the power consumption as well as cost saving and improve the response time [7]

Kepi Zhang, Tong Wu and all have proposed algorithm which focus on the energy saving issue for virtual machine (VM) selections on an overloaded host in a cloud computing environment [11]. They analyze the energy influencing factors during a VM migration, then design energy efficient VM selection algorithms based on greedy algorithm and dynamic programming method. It reduces energy consumption using proposed method.

GarimaBatra, Harshita Singh and all have proposed algorithm where many virtual machines are created on one physical machine (Host) [12]. Another version of VM placement is presented by them that uses the best fit technique to allocate VMs onto physical machine, since the effects on power utilization and memory sharing for various virtual machinephysical machine combinations while making the decision. The consideration of these two factors makes the presented algorithm more efficient than algorithms that are oblivious to these factors as well as those which consider either of them.

LI Hongyou, WANG Jiangyong and all have proposed two algorithms called the Energy-aware Scheduling algorithm using Workload-aware Consolidation Technique (ESWCT) and the Energy- aware Live Migration algorithm using Workload-aware Consolidation Technique (ELMWCT) [13]. They focus on multiple resources for scheduling like RAM, CPU and network bandwidth and result show reduced energy consumption than one dimensional resource.

\section{Proposed DVIMIRB method}

\section{Dynamic VIM Creation}

In this phase, virtual machines are classified in three categories: compute intensive, data intensive and mix load and Allocate task based on task category.Compute intensive task is assigned to compute intensive VM, data intensive task is assigned to data intensive $\mathrm{VM}$ and so on.

The number of VM should be optimum so that it can maintain the quality of service parameters and consumes less energy. If number of VM is more, it consumes more power and if number of VM is less, it will violate the Service Level Agreement (SLA) and degrade the performance of the system. Hence, when more than $70 \%$ of VM is overloaded, scheduler will create a newVM. This condition is represented by equation 5 .

Number of overloaded $V M>0.7 *$ total $V M$ create a new $V M$ (5)

Once the task completes its execution, virtual machine will be freed, therefore some of the virtual machines need to be switched off to get optimum results. Hence

Number of overloadedVM $<0.3 *$ totalVM

Therefore, a virtual machine is overloaded if it holds equations (7), (8) and (9) true:

$T U_{M}>T H_{M}$
$T U_{R}>T H_{R}$

(8)

$T U_{B}>T H_{B}$

Here, $T U_{M}$ is representing the MIPS actual utilization and $T H_{M}$ is representing the threshold of MIPS. $T U_{R}$ is actual RAM utilization and $T H_{R}$ is threshold value of RAM utilization. $T U_{B}$ is actual Bandwidth utilization and $T H_{B}$ is threshold value of Bandwidth utilization. Whenever the actual utilization of MIPS, memory and bandwidth by a virtual machine is exceeding the threshold value of MIPS, memory and bandwidth of virtual machine, then virtual machine is considered to be overloaded.

In this paper,we propose virtual machine migration technique for minimizing the power consumption. Power is consumed by host so in this technique we try to minimize total running machine by switching off some of the half utilized host. As shown in algorithm 1 load balancer check utilization of host in processing time interval and calculate host utilization from If host utilization is more than upper threshold than some of the vm will be migrated from that host to a host which is not over utilized. Here host will overloaded by any one of this over utilized resource it can be memory, MIPS or bandwidth. So here we will find a target host which have lower utilization of that parameter and create VM on that host. If resources of host are under loaded than migrate all VM from the host and switch off that host.

$\mathrm{U}_{\mathrm{Ram}}=$ RamUtilization,

$\mathrm{U}_{\text {mips }}=$ Mipsutilization,

$\mathrm{U}_{\text {Bandwidth }}=$ Bandwidth Utilization

Algorithm 1: Calculate host utilization

Input: $\mathrm{U}_{\mathrm{Ram}}=$ ramUtilization, $\mathrm{U}_{\text {mips }}=$ mipsutilization, $\mathrm{U}_{\text {Bandwidth }}=$ bwUtilization

Output:hostutilization,

For Host $h$ from Hostlist

If $\mathrm{U}_{\text {Ram }}$ is higher than $\mathrm{U}_{\text {mips }}$ and $\mathrm{U}_{\text {Bandwidth }}$ then

$\mathrm{U}=\mathrm{U}_{\text {Ram }} * \mathrm{U}_{\text {Ram }}+\left(1-\mathrm{U}_{\text {Ram }}\right) *\left(\mathrm{U}_{\text {mips }}+\mathrm{U}_{\text {Bandwidth }}\right) / 2$

Endif

Elseif $\mathrm{MU}$ is higher than RU and BU then

Else

$$
\mathrm{U}=\mathrm{U}_{\text {mips }} * \mathrm{U}_{\text {mips }}+\left(1-\mathrm{U}_{\text {mips }}\right) *\left(\mathrm{U}_{\text {Ram }}+\mathrm{U}_{\text {Bandwidth }}\right) / 2
$$

$$
\mathrm{U}=\mathrm{U}_{\text {Bandwidth }} * \mathrm{U}_{\text {Bandwidth }}+\left(1-\mathrm{U}_{\text {Bandwidth }}\right) *\left(\mathrm{U}_{\text {mips }}+\mathrm{U}_{\text {Ram }}\right) / 2
$$

\section{VM Migration}

This approach is focus on migration of virtual machine where we have to identify the host having higher workload then we will find the vm for migration of vm. As per the Algorithm 2 it will identify vm from list of vms to migrate from overloaded host to other host. for selection of vm it identify the maximum utilization of parameters like ram, mips, bandwidth and find the maximum utilization of that parameters. Then migrate the vm which has higher utilization of maximum utilized parameter. Example like if host is overloaded and total utilization is $89 \%$ and in this host ram utilization is like $90 \%$ which most and ram, mips, and bw then it will select the vm from that host which utilize maximum ram and migrate it to another host.

Algorithm 2: SelectingVM for Migration

$U_{\text {ram }}$ defineutilization of Ram, $U_{\text {mips }}$ define utilization of mips and $U_{b w}$ define utilization of bandwidth, $P$ defined for physical machine

VMtoMigrate

NULL

Vmid

NULL

$\operatorname{Max}$

0

If $\left(\mathrm{U}_{\text {ram }}>\mathrm{U}_{\text {mips }}\right)$ and If $\left(\mathrm{U}_{\mathrm{ram}}>\mathrm{U}_{\mathrm{bw}}\right)$

For each $\mathrm{VM}_{\mathrm{i}}$ in overloaded $\mathrm{PM}$

Find vm which utilized maximum bandwidth and assigned it 
toVM_Migrate for migration

End If

Else If $\left(\mathrm{U}_{\text {mips }}>\mathrm{U}_{\mathrm{bw}}\right)$

For each VM $v_{i}$ in overloaded PM

Find vm which utilized maximum bandwidth and assigned it toVM_Migrate for migration

End Else if

Else

For each $\mathrm{VM} \mathrm{v}_{\mathrm{i}}$ in overloaded $\mathrm{P}$

Find vm which utilized maximum bandwidth and assigned it toVM_Migrate for migration

End Else

\section{Finding Host forVIM placement}

As per algorithm 3 it select the host which is not overloaded and check that after placement of vm there must not be over utilization of host as well as any parameters.

\section{Algorithm 3: Selecting Physical Machine for VM Placement}

issuitablehost $(p, v m)$ return true if it has enough resource to create vm on $p$

Selected_P NULL

For each PM $\mathrm{p}$ inPMList

If (issuitablehost ( $p, v m)$ )

AllocateVM(p,vm)

$/ / \mathrm{vm}$ is created on $\mathrm{pm}$

If(isoverloaded (pm))

Deallocate (p,vm) // if host is overload then deallocate vm

End If

Else

Break

End if

End for

\section{Experiment and Results}

We implemented algorithm in cloudsim with $50 \mathrm{VM}$ and 10 host. we compare proposed DVMMRB method with threshold based method and ratio based method.

\section{Table 2: Energy consumptions}

\begin{tabular}{|c|c|c|c|}
\hline no. of cloudlet & $\begin{array}{c}\text { Threshold } \\
\text { Method }\end{array}$ & $\begin{array}{c}\text { Ratio Based } \\
\text { Method }\end{array}$ & DVMMRB \\
\hline 500 & 125.75 & 117.25 & 115.23 \\
\hline 600 & 134.54 & 131.12 & 129.08 \\
\hline 700 & 180.45 & 165.56 & 160.47 \\
\hline 800 & 184.34 & 170.34 & 165.32 \\
\hline 900 & 185.12 & 175.56 & 168.85 \\
\hline 1000 & 195.65 & 182.43 & 174.62 \\
\hline
\end{tabular}

\section{Energy Consumption}

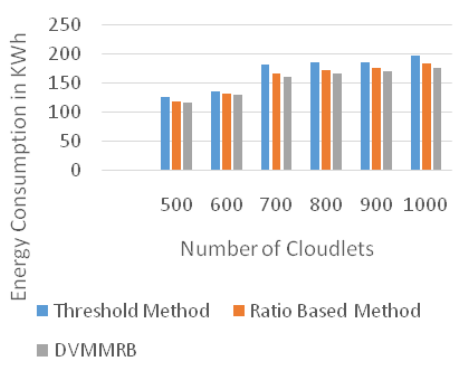

Figure 1 Energy Consumption Comparison
Table 3:VM Migration

\begin{tabular}{|c|c|c|c|}
\hline $\begin{array}{c}\text { no. of } \\
\text { cloudlet }\end{array}$ & $\begin{array}{c}\text { Threshold } \\
\text { Method }\end{array}$ & $\begin{array}{c}\text { Ratio Based } \\
\text { Method }\end{array}$ & DVMMRB \\
\hline 500 & 140 & 123 & 120 \\
\hline 600 & 153 & 132 & 128 \\
\hline 700 & 185 & 165 & 156 \\
\hline 800 & 195 & 170 & 167 \\
\hline 900 & 208 & 190 & 182 \\
\hline 1000 & 227 & 210 & 198 \\
\hline
\end{tabular}

\section{VM Migration}

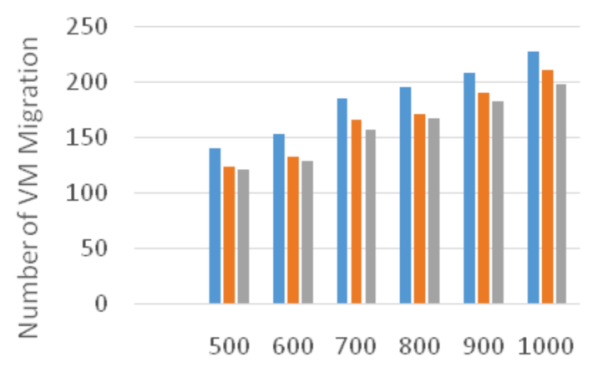

Number of Cloudlets

- VM Migration VM Migration

\section{Figure 2VIM Migration Comparison}

Given simulation results shows improvements in reducing Energy Consumption. If we consider the existing approach then it uses static threshold based method to identify overloaded host. But contradictory in this proposed approached, we have considered 3 parameters, i.e. MIPS, RAM and Bandwidth, to utilize CPU, Memory and Network in the existing cloud infrastructure.

Add-on, if we compare both the approaches, existing approach [9] with our proposed approach, then it shows a clear distinctions for Energy consumption. In the existing approach, as we are increasing No. of cloudlets, Energy consumption is gradually increases. But when we compare the Energy consumption of existing work with proposed approach, then my system works on very low Energy consumption. And as per cloud computing cost calculation, energy consumption is directly proportional to total cost. Hence this system will also reduce the total cost during execution. As well, low energy consumption will reduce the emission of Carbon dioxide.

\section{CONCLUSION}

We analyzed energy consumption on different parameters consideration by applying our proposed approach. The proposed DVMMRB provides better energy optimization that is done in cloudsim tool. Energy consumption results have been compared with existing threshold based and ratio based approach and the results shows that DVMMRB outperforms the existing methods. Similarly, the proposed DVMMRB method have been compared with existing threshold based and ratio based method in terms of VM migration. The results shows that proposed DVMMRB outperforms among all algorithms. But still there are some possible areas in which future investigation is needed.

\section{REFERENCES}

1. M. H. Kumar, "Energy Efficient Task Scheduling for Parallel Workflows in Cloud Environment,"pp. 1298-1303,2014.

2. T. V. T. Duy, Y. Sato, and Y. Inoguchi, "Performance evaluation of a green scheduling algorithm for energy savings in cloud computing," Proc. 2010 IEEE Int. Symp. Parallel Distrib. Process. Work. Phd Forum, IPDPSW 2010, no. September 2015,pp. 1-8,2010.

3. N. Kim, J. Cho, and E. Seo, "Energy-credit scheduler: An energy-aware virtual machine scheduler for cloud systems," Futur. Gener. Comput. Syst., vol. 32, no. 1,pp. 128-137,2014. 
4. J. Baliga, R.W. a Ayre, K. Hinton, and R. S. Tucker, "Green Cloud Computing: Balancing Energy in Processing, Storage and Transport,"Proc.IEEE, 2010.

5. L. Luo, W. Wu, D. Di, and F. Zhang, "A resource scheduling algorithm of cloud computing based on energy efficient optimization methods," Green Comput. Conf., no.July 2007,pp. 0-5,2012.

6. Y.-J. Chiang, Y.-C. Ouyang, and C.-H. R. Hsu, "An Efficient Green Control Algorithm in Cloud Computing for Cost Optimization," IEEE Trans. Cloud Comput.,vol.3,no.2,pp. 145-155,2015.

7. A. P. M. De La, F.Vigliotti, and D. Macêdo, "Energy-Efficient Virtual Machines Placement,"'Sbrc 2014,pp. 17-30,2014.

8. Bhaskar Prasad, Eunmi Choi and Ian Lumb, "A Taxonomy, Survey, and Issues of Cloud Computing Ecosystems", Springer International Conference on Computational Intelligence and Computing Research, ISBN: 978-184996240-7,2010, pp:21-46.

9. Anton Beloglazov,Jemal Abawajy,Rajkumar Buyya, "Energy Efficent resource allocation heustricls for efficient management of data center for cloud computing"-Science direct,Vol.28,PP. 755-768,2014

10. Anton Beloglazov and Rajkumar Buyya," Energy Efficient Resource Management in Virtualized Cloud Data Centers" 2010 10th IEEE/ACM International Conference onCluster, cloud and Grid Computing, 44,989100 